\title{
SOME ANALOGUES OF KNOPP'S CORE THEOREM
}

\author{
I.J. MADDOX \\ Department of Pure Mathematics \\ Queen's University of Belfast \\ Belfast BT7 1NN \\ Northern Ireland
}

(Received Apri1 23, 1979)

ABSTRACT. Inequalities between certain functionals on the space of bounded real sequences are considered. Such inequalities being analogues of the classical theorem of Knopp on the core of a sequence. Also, a result is given on infinite matrices of bounded linear operators acting on bounded sequences in a Banach space.

KEY WORDS AND PHRASES. Core theorem, Functionals on the bounded sequences, Infinite matrices.

1980 MATHEMATICS SUBJECT CLASSIFICATION CODES. $40 \mathrm{C} 05,40 J 05$.

1. INTRODUCTION.

For a real sequence $x=\left(x_{k}\right)$ we write

$$
\ell(x)=1 \text { im } \inf x_{k}, L(x)=1 \text { im } \sup x_{k},
$$




$$
\begin{aligned}
& y(x)=\lim \inf \frac{x_{1}+x_{2}+\ldots+x_{k}}{k}, \\
& Y(x)=\lim \sup \frac{x_{1}+x_{2}+\ldots+x_{k}}{k}, \\
& w(x)=\inf \{L(x+z): z \in b s\}, \\
& S(x)=\sup x_{k},|| x||=\sup \left|x_{k}\right|, \\
& p(x)=\lim \sup \left|x_{k}\right|, q(x)=1 \text { im inf }\left|x_{k}\right| .
\end{aligned}
$$

In the definition of $w$ we use bs to denote the space of all 'bounded series', more precisely:

$$
\text { bs }=\left\{z: \sup _{n}\left|\sum_{k=1}^{n} z_{k}\right|<\infty\right\} .
$$

If $A=\left(a_{n k}\right)$ is an infinite matrix of real, or complex, numbers, we write

$$
\mathrm{Ax}=\left(\sum \mathrm{a}_{\mathrm{nk}} \mathrm{x}_{\mathrm{k}}\right)
$$

where all sums are from $k=1$ to $k=\infty$, unless otherwise indicated.

Let $X$ be a Banach space with norm $\|x\|$ and let $B(X)$ be the Banach space of bounded linear operators on $\mathrm{X}$ into $\mathrm{X}$ with the usual operator norm. The space of bounded $X$-valued sequences is denoted by $\ell_{\infty}(x)$, with $\|x\|=\sup _{n}|| x_{n} \|$, for each $x \in \ell_{\infty}(X)$. By $c(X)$ we denote the space of convergent $X$-valued sequences.

If $G$ and $H$ are real functionals on $\ell_{\infty}(X)$, and $M \geq 0$ is a real number, then $G \leq M H$ means that $G(x) \leq M H(x)$ for all $x \in \ell_{\infty}(X)$.

In connection with a real matrix A, we shall write, for example, LA $\leq \mathrm{L}$ to mean that Ax exists for all $x \in \ell_{\infty}(R)$ and that $L(A x) \leq L(x)$ for all $\mathbf{x} \in \ell_{\infty}(\mathrm{R})$.

Devi [1] refers to the result that: "LA $\leq L$ if and only if $A$ is regular and almost positive", as Knopp's core theorem, and refers to Cooke [2] for the proof. Strictly speaking the result as stated does not seem to be given 
by Cooke, though the ingredients for a proof are there. In Section 2 below we indicate, for completeness, a brief proof of the result.

Using Knopp's core theorem, Devi [1] proves that LA $\leq w$ if and only if A is strongly regular and almost positive. To say that $A$ is strongly regular is to say that $A$ is regular and

$$
\Sigma\left|a_{n k}-a_{n, k+1}\right| \rightarrow 0(n \rightarrow \infty) .
$$

In Section 2 we prove that $\mathrm{LA} \leq \mathrm{y}$ is impossible, and that $\mathrm{LA} \leq \ell$ is impossible. Also, necessary and sufficient conditions are given for $p A \leq q$.

In Section 3 we give a theorem involving pA for bounded sequences from $X$, and infinite matrices $\left(A_{n k}\right)$ from $B(X)$.

2. REAL BOUNDED SEQUENCES.

We first give exact conditions for $L A \leq L$, as mentioned in Section 1 .

THEOREM 1. LA $\leq \mathrm{L}$ if and only if $\mathrm{A}$ is regular and

$$
\Sigma\left|a_{n k}\right| \rightarrow 1(n \rightarrow \infty) \text {. }
$$

PROOF. For the necessity, let $x \in c(R)$. Then $\ell(x)=L(x)=1$ im $x_{n}$ and $L(A(-x)) \leq L(-x)$, whence

$$
\lim x_{n} \leq \ell(A x) \leq L(A x) \leq L(x)=\lim x_{n}
$$

and so $A x \in c(R)$ with $\lim (A x)_{n}=\lim x_{n}$, which implies $A$ is regular. By the Silverman-Toeplitz theorem, see e.g. Maddox [3], p.165, it follows that

$$
\begin{aligned}
& H=1 \text { im } \sup _{n} \Sigma\left|a_{n k}\right|<\infty, \\
& \sum a_{n k} \rightarrow 1(n \rightarrow \infty),
\end{aligned}
$$




$$
a_{n k} \rightarrow 0(n \rightarrow \infty \text {, each fixed } k)
$$

From (2.2), (2.4), e.g. Agnew [4], there exists y $\epsilon \ell_{\infty}(R)$ such that $\|y\|=1$ and $L(A y)=H$. Hence, by $(2.3)$,

$$
1 \leq 1 \mathrm{im} \inf _{\mathrm{n}} \Sigma\left|\mathrm{a}_{\mathrm{nk}}\right| \leq 1 \mathrm{im} \sup _{\mathrm{n}} \sum\left|\mathrm{a}_{\mathrm{nk}}\right| \leq \mathrm{L}(\mathrm{y}) \leq\|\mathrm{y}\| \leq 1,
$$

which implies (2.1).

For the sufficiency, let $x \in \ell_{\infty}(R)$, $A$ be regular and let (2.1) hold. If m $>1$ then

$$
\sum a_{n k} x_{k} \leq|| x|| \sum_{k<m}\left|a_{n k}\right|+\left(\sup _{k \geq m} x_{k}\right) \Sigma\left|a_{n k}\right|+|| x|| \Sigma\left(\left|a_{n k}\right|-a_{n k}\right) .
$$

Applying the operator $\lim _{m} \lim \sup _{n}$ we obtain $L(A x) \leq L(x)$, which completes the proof.

THEOREM 2. We have, on $\ell_{\infty}(\mathrm{R})$,

$$
\ell \leq \mathrm{y} \leq \mathrm{Y} \leq \mathrm{w} \leq \mathrm{L} \leq \mathrm{S} \leq\|\cdot\| \text {. }
$$

PROOF. By Theorem 1 , letting A be the $(C, 1)$ matrix, we have $\ell \leq \ell A$, i.e. $\ell \leq \mathrm{y}$. It is trivial that $\mathrm{y} \leq \mathrm{Y}$.

Now take $x \in \ell_{\infty}(R)$ and $z \in$ bs. Then

$$
\frac{1}{k} \sum_{i=1}^{k} x_{i}=\frac{1}{k} \sum_{i=1}^{k}\left(x_{i}+z_{i}\right)+\varepsilon_{k},
$$

where $\lim \varepsilon_{k}=0$. Taking $\lim \sup _{k}$ in (2.5), and applying Theorem 1 with $A=(c, 1)$, we get $Y(x) \leq L(x+z)$, whence $Y \leq w$ by the definition of $w$.

Since $\theta=(0,0,0, \ldots) \in$ bs it is immediate that $w \leq L$, and the remaining inequalities are trivial. 
The facts that $\mathrm{LA} \leq \mathrm{y}$, and $\mathrm{LA} \leq \ell$ are impossible are special cases of the following result.

THEOREM 3. Let $B$ be any regular almost positive matrix. Then there is no matrix A such that $L A \leq \ell B$.

PROOF. Suppose, if possible, there exists such an A. Theorem 1 implies $\mathrm{LB} \leq \mathrm{L}$, and so $\mathrm{LA} \leq \ell \mathrm{B} \leq \mathrm{LB} \leq \mathrm{L}$, whence $\mathrm{A}$ is regular.

By the theorem of Steinhaus, see e.g. Cooke [2], p.75, there exists $z \in \ell_{\infty}(R)$ such that $\ell(A z)<L(A z)$. Since $L A \leq L B$ we have $\ell(B z) \leq \ell(A z)$, and so

$$
\ell(B z)<L(A z) \leq \ell(B z),
$$

a contradiction. This proves the theorem.

The statement prior to Theorem 3 follows on taking B to be either the $(C, 1)$ matrix, or the unit matrix.

THEOREM 4. The following are equivalent:

$$
\mathrm{pA} \leq \mathrm{q},
$$

A maps bounded sequences into null sequences,

$$
\Sigma\left|a_{n k}\right| \rightarrow 0(n \rightarrow \infty)
$$

PROOF. The equivalence of (2.7) and (2.8) is we11-known, see e.g. Maddox [3], p.169. We shall prove that $(2.6)$ is equivalent to $(2.8)$. If (2.8) holds then, for all $x \in \ell_{\infty}(R)$, $\lim \sup _{\mathrm{n}}\left|\Sigma_{\mathrm{ak}_{\mathrm{k}}} \mathrm{x}_{\mathrm{k}}\right|=0$, 
which implies (2.6). Conversely, let (2.6) hold. Then $\sum a_{n k} x_{k}$ is bounded on the Banach space $\ell_{\infty}(R)$ whence $\sup _{n} \sum\left|a_{n k}\right|<\infty$ by the Banach-Steinhaus theorem. Also, choosing $x_{k}=1, x_{n}=0$ otherwise, we must have (2.4).

Suppose, if possible, that $\lim \sup _{n} \Sigma\left|a_{n k}\right|=d>0$. Choose $m(1)>1$ such that $\left|a_{m(1) 1}\right|<d / 10$ and

$$
|\Sigma| a_{m(1) k}|-d|<d / 10
$$

Define $k(1)=1$ and choose $k(2)>2+k(1)$ such that

$$
\sum_{k(2)}^{\infty}\left|a_{m(1) k}\right|<d / 10
$$

Next choose $m(2)>m(1)$ such that

$$
\sum_{1}^{k(2)}\left|a_{m(2) k}\right|<d / 10,|\Sigma| a_{m(2) k}|-d|<d / 10,
$$

and choose $k(3)>2+k(2)$ such that

$$
\underset{k(3)}{\infty}\left|a_{m(2) k}\right|<d / 10
$$

Proceeding inductively we now define a sequence $\mathrm{x}$ by

$$
\begin{aligned}
& x_{k}=\operatorname{sgn} a_{m(r) k} \text { for } k(r)<k<k(r+1), r \geq 1 \text {, } \\
& x_{k}=0 \quad \text { for } k=k(r+1), \quad r \geq 0 \text {. }
\end{aligned}
$$

Then ||$x|| \leq 1$ and $\lim \inf \left|x_{k}\right|=0$, so (2.6) implies

$$
\mathrm{p}(\mathrm{Ax})=0
$$

But for $m=m(r)$, with $r>1$, we have 


$$
\left|\Sigma \mathrm{a}_{\mathrm{mk}} \mathrm{x}_{\mathrm{k}}\right|>\Sigma_{1}\left|\mathrm{a}_{\mathrm{mk}}\right|-\mathrm{d} / 5
$$

where $\Sigma_{1}$ denotes a sum over $k(r)<k<k(r+1)$. Also, we have

$$
\left|\Sigma_{1}\right| a_{m k}|-d|<3 d / 10
$$

and so

$$
\left|\sum \mathrm{a}_{\mathrm{mk}} \mathrm{x}_{\mathrm{k}}\right|>\mathrm{d}-3 \mathrm{~d} / 10-\mathrm{d} / 5=\mathrm{d} / 2 \text {. }
$$

Since (2.10) holds for infinitely many $m$ it follows that

$$
\mathrm{p}(\mathrm{Ax}) \geq \mathrm{d} / 2 \text {. }
$$

But (2.11) contradicts (2.9), so $\mathrm{d}=0$, and the proof is complete.

3. BOUNDED SEQUENCES IN A BANACH SPACE.

$$
\begin{aligned}
& \text { Define, for each } x=\left(x_{k}\right) \in \ell_{\infty}(X), \\
& G(X)=1 \text { im sup }|| x_{k}||, \\
& H(x)=\inf \{G(x+z): z \in b s(x)\},
\end{aligned}
$$

where

$$
\mathrm{bs}(\mathrm{X})=\left\{\mathrm{z}: \sup _{\mathrm{n}}|| \sum_{\mathrm{k}=1}^{\mathrm{n}} \mathrm{z}_{\mathrm{k}} \|<\infty\right\} \text {. }
$$

Thus $G$ and $H$ many be regarded as the Banach space analogues of $\mathrm{p}$ and $\mathrm{w}$ which appeared earlier.

By $\mathrm{GA} \leq \mathrm{MH}$ we mean that $\mathrm{G}(\mathrm{Ax}) \leq \mathrm{MH}(\mathrm{x})$ for all $\mathrm{x} \in \ell_{\infty}(\mathrm{X})$, where

$$
A x=\left(\sum A_{n k} x_{k}\right)
$$

with $A_{n k} \in B(X)$. 
It is clear that $\mathrm{bs}(\mathrm{X}) \subset \ell_{\infty}(\mathrm{X})$, and that $0 \leq \mathrm{H}(\mathrm{x}) \leq \mathrm{G}(\mathrm{x})<\infty$ for a11 $\mathbf{x} \in \ell_{\infty}(\mathrm{X})$

Also, since $-x \in b s(X)$ whenever $x \in b s(X)$ we have that

$$
\mathrm{H}(\mathrm{x})=0 \text { on } \mathrm{bs}(\mathrm{X}) \text {. }
$$

In the following theorem we need the ideas of the group norm of a sequence $\left(B_{k}\right)$ from $B(X)$, see e.g. Lorentz and Macphail [5]:

$$
\left\|\left(B_{k}\right)||=\sup || \sum_{k=1}^{n} B_{k} x_{k}\right\|
$$

where the supremum is over $n \geq 1$ and $x_{k}$ in the closed unit sphere of $x$.

We write

$$
\mathrm{R}_{\mathrm{nm}}=\left(\mathrm{A}_{\mathrm{nm}}, \mathrm{A}_{\mathrm{n}, \mathrm{m}+1}, \ldots\right)
$$

for the mth tail of the $n t h$ row of $A=\left(A_{n k}\right)$. Also, we define $\Delta \mathrm{A}_{\mathrm{nk}}=\mathrm{A}_{\mathrm{nk}}-\mathrm{A}_{\mathrm{n}, \mathrm{k}+1}$, and

$$
\Delta R_{n m}=\left(\Delta A_{n, m}, \Delta A_{n, m+1}, \ldots\right)
$$

We now prove

THEOREM 5. Let $M \geq 0$. Then GA $\leq$ MH if and only if

$$
\begin{gathered}
A_{n k} \rightarrow 0(n \rightarrow \infty \text {, each } k) \\
|| R_{n 1} \mid<\infty \text { and }|| R_{n m}|| \rightarrow 0(m \rightarrow \infty \text {, each } n) \\
1 \text { im } 1 \text { im } \sup _{n}|| R_{n m}|| \leq M \\
\lim _{m} \lim \sup _{n}|| \Delta R_{n m}||=0
\end{gathered}
$$

PROOF. We remark that, in (2.12), the convergence refers to the topology 
of pointwise convergence.

For the sufficiency, let $x \in \ell_{\infty}(X)$, and $z \in b s(X)$. By Maddox $[6$, THEOREM 1] the conditions (2.12), (2.13), (2.14) imply GA $\leq M G$, whence $G A(x+z) \leq M G(x+z)$, and so

$$
G(A x) \leq M G(x+z)+G(A z) .
$$

Now

$$
\underset{k=1}{r} A_{n k} z_{k}=A_{n r} s_{r}+\sum_{k=1}^{r-1} \Delta A_{n k} s_{k},
$$

where $s_{k}=z_{1}+z_{2}+\ldots+z_{k}$. Since $\left\|A_{n r} s_{r}\right\| \leq\left\|A_{n r}\right\|\left\|s_{r}\right\|$, and since $s \in \ell_{\infty}(X)$, it follows from (2.13) and (2.17) that, for each $n$,

$$
\sum \mathrm{A}_{\mathrm{nk}} \mathrm{z}_{\mathrm{k}}=\Sigma \Delta \mathrm{A}_{\mathrm{nk}} \mathrm{s}_{\mathrm{k}}
$$

By Maddox [6, COROLLARY to THEOREM 1], the conditions (2.12) - (2.15) imply that $\triangle A: \ell_{\infty}(X) \rightarrow c_{0}(X)$, where $c_{0}(X)$ denotes the null X-valued sequences. Hence from (2.18) we have $G(A z)=0$, whence (2.16) yields $G(A x) \leq M G(x+z)$. It follows that $G(A x) \leq M H(x)$, which proves the sufficiency.

For the necessity, if $G A \leq M H$ then $G A \leq M G$ so that (2.12) - (2.14) hold by Maddox [6, THEOREM 1$]$.

Now take any $y \in \ell_{\infty}(x)$ and define $x_{1}=y_{1}, x_{2}=y_{2}-y_{1}, \ldots$, so that

$$
x_{1}+x_{2}+\ldots+x_{n}=y_{n} \text {. }
$$

Thus $x \in b s(x)$ and

$$
\Sigma_{\mathrm{A}_{\mathrm{nk}} \mathrm{x}_{\mathrm{k}}}=\Sigma \Delta \mathrm{A}_{\mathrm{nk}} \mathrm{y}_{\mathrm{k}} .
$$


Hence $G(\triangle A y)=G(A x) \leq M H(x)=0$, since $H(x)=0$ on $\ell_{\infty}(X)$. Consequently, $G(\Delta A y)=0$ on $\ell_{\infty}(X)$, which implies $\Delta A: l_{\infty}(X) \rightarrow c_{0}(X)$, whence (2.15) holds by [6, COROLLARY TO THEOREM 1]. This proves the theorem.

\section{REFERENCES}

1. Devi, S.L. Banach limits and infinite matrices, J. London Math. Soc. $\underline{12}$ (1976) 397-401.

2. Cooke, R.G. Infinite matrices and sequence spaces, Macmi11an, 1950.

3. Maddox, I.J. Elements of Functional Analysis, Cambridge University Press, 1970.

4. Agnew, R.P. Abel transforms and partial sums of Tauberian series, Annals of Math. 50 (1949) 110-117.

5. Lorentz, G.G. and Macphail, M.S. Unbounded operators and a theorem of A. Robinson, Trans. Roy. Soc. Canada, Sec. III (3) 46 (1952) 33-37.

6. Maddox, I.J. Matrix maps of bounded sequences in a Banach space, Proc. American Math. Soc. 63 (1977) 82-86. 


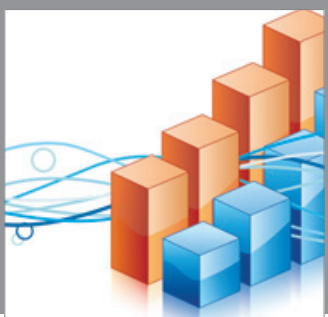

Advances in

Operations Research

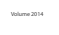

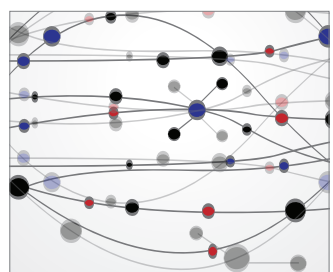

\section{The Scientific} World Journal
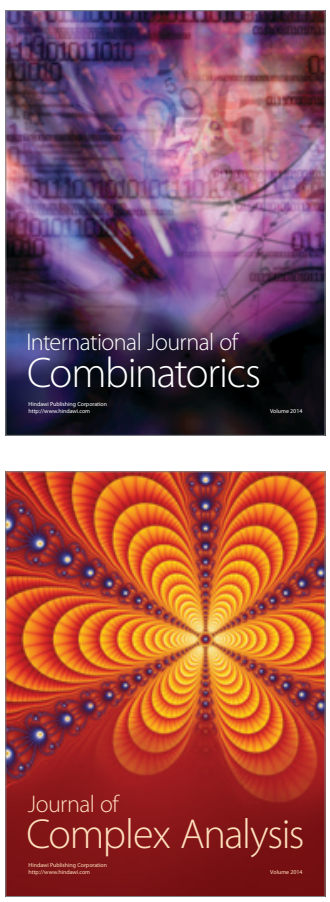

International Journal of

Mathematics and

Mathematical

Sciences
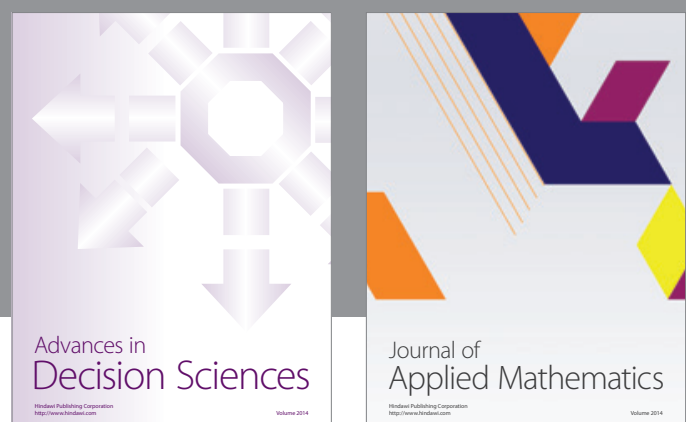

Journal of

Applied Mathematics
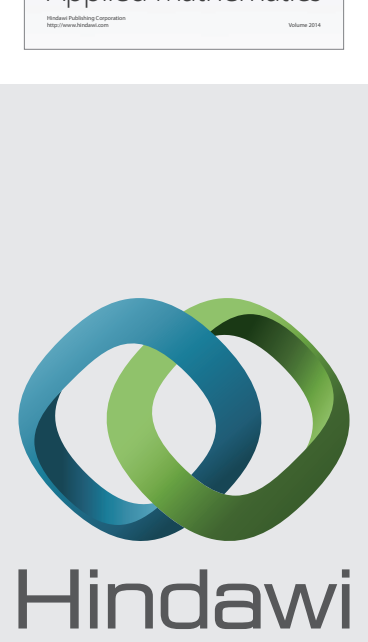

Submit your manuscripts at http://www.hindawi.com
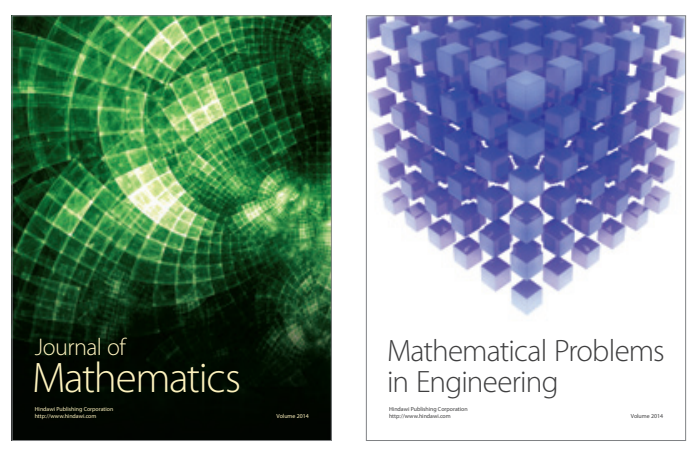

Mathematical Problems in Engineering
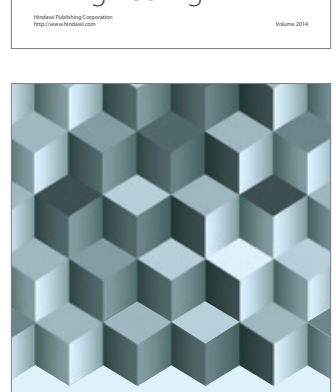

Journal of

Function Spaces
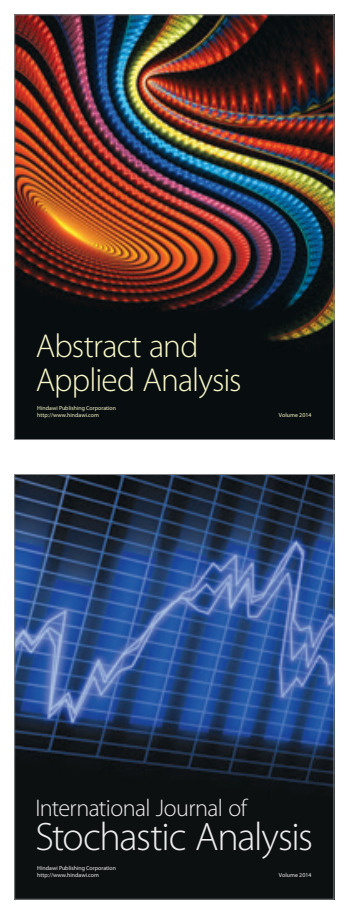

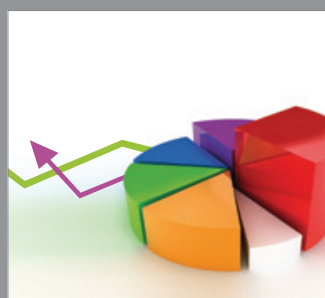

ournal of

Probability and Statistics

Promensencen
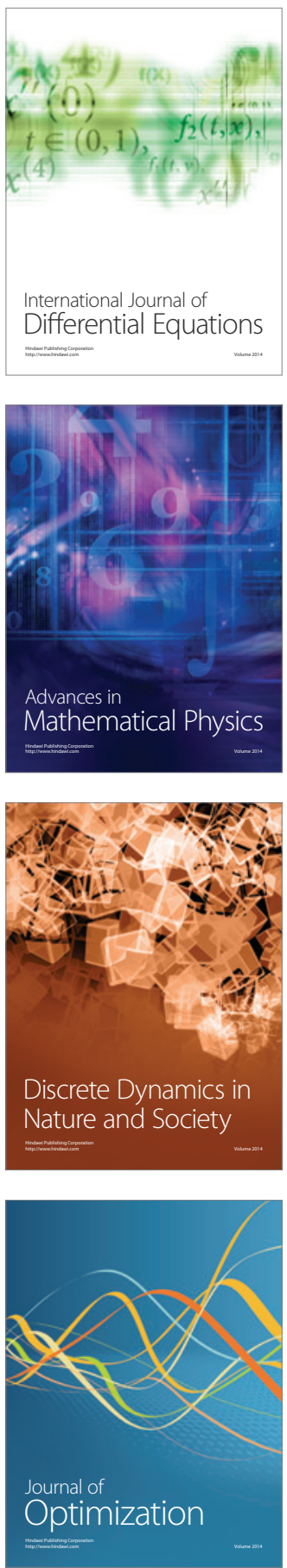